\title{
5.5 A Femoral 'Stealing' Effect: Presence of Collateral Retrograde Blood Flow Redistribution in the Femoral Bifurcation During Reactive Hyperemia
}

\author{
Jason $\mathrm{Au}^{1,2, *}$, Robert Amelard ${ }^{2}$, Billy Yiu' ${ }^{1,2}$, Hassan Nahas ${ }^{1,2}$, Richard Hughson ${ }^{2}$, Alfred Yu ${ }^{1,2}$ \\ ${ }^{1}$ University of Waterloo, Waterloo, Canada \\ ${ }^{2}$ Schlegel-University of Waterloo Research Institute for Aging, Waterloo, Canada
}

\section{ABSTRACT}

Background \& Purpose: The femoral bifurcation houses complex flow recirculation that may be impacted by downstream peripheral resistance; however, conventional ultrasound is unable to quantify multi-directional flow to study these phenomena. The purpose of this study was to examine the multi-directional behavior of blood in the femoral bifurcation during reactive hyperemia (RH) using vector flow imaging.

Methods: Nine healthy adults ( $25 \pm 4$ years; 5 men) underwent high-frame-rate ultrasound imaging of their right femoral bifurcation at rest, and during the first cardiac cycle of RH after 5-min of $200 \mathrm{mmHg}$ calf occlusion. Multi-angle Doppler ultrasound was used to qualitatively describe vector blood flow at $750 \mathrm{fps}$ [1]. The bifurcation region was isolated for blood vector dispersion analysis, an index of flow directionality [2].

Results: Blood flow during RH was characterized by near-constant anterograde blood flow to the superficial femoral artery (SFA), adjacent to low/oscillatory flow immediately proximal to the deep femoral artery (DFA) (Figure 1). We observed collateral blood flow redistribution in 3/9 participants, where blood from the DFA appeared to re-enter the SFA during the retrograde flow period. Bifurcation vector dispersion was reduced from baseline ( $0.53 \pm 0.08$ vs $0.67 \pm 0.09 ; p<0.01)$, but not eliminated, due to preservation of DFA flow reversal.

Conclusion: We demonstrate the presence of distinct, and potentially interacting, blood flow streams within the femoral bifurcation during RH. These observations were made possible by the emerging vector flow imaging technique to study complex hemodynamics in vivo, and suggest novel mechanisms by which blood is distributed to vascular beds, in extreme flow profiles.
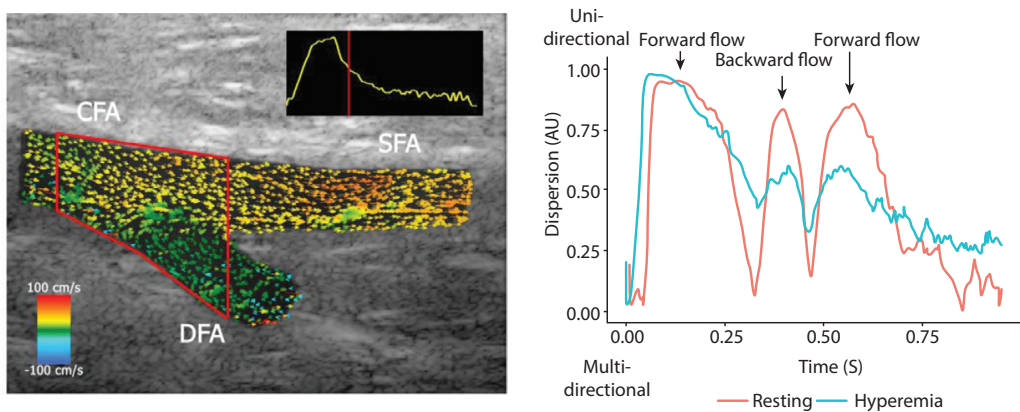

Figure 1 Left: Representative example (31 yr old man) of vector projectile imaging of the femoral bifurcation at endsystole; vectors within the red region of interest were extracted to calculate vector dispersion. Right: Representative example of blood vector dispersion at rest and during hyperemia during a single cardiac cycle; values closer to 0 indicate greater dispersion.

\section{REFERENCES}

[1] Yiu BYS, Yu ACH. Least-squares multi-angle doppler estimators for plane-wave vector flow imaging. IEEE Trans Ultrason Ferroelectr Freq Control 2016;63:1733-44.

[2] Pedersen MM, Pihl MJ, Haugaard P, Hansen KL, Lange T, Lönn L, et al. Novel flow quantification of the carotid bulb and the common carotid artery with vector flow ultrasound. Ultrasound Med Biol 2014;40:2700-6.

(C) 2019 Association for Research into Arterial Structure and Physiology. Publishing services by Atlantis Press International B.V. This is an open access article distributed under the CC BY-NC 4.0 license (http://creativecommons.org/licenses/by-nc/4.0/). 